\title{
Evaluation of fracture toughness of small volumes by means of cube-corner nanoindentation
}

\author{
N. Cuadrado*a, D. Casellas ${ }^{a, b}$, M. Anglada ${ }^{c}$, E. Jiménez-Piquéc \\ ${ }^{a}$ Fundació CTM Centre Tecnològic; Avda. de les Bases de Manresa 1, 08242 Manresa, Spain. \\ ${ }^{\mathrm{b}}$ Department of Materials Science and Metallurgical Engineering, EPSEM, Universitat \\ Politècnica de Catalunya, Avda. de les Bases de Manresa 61, 08242 Manresa, Spain \\ ${ }^{c}$ Department of Materials Science and Metallurgical Engineering, ETSEIB, Universitat \\ Politècnica de Catalunya, Avda. Diagonal 647, 08028 Barcelona, Spain
}

Corresponding author*: e-mail: nuria.cuadrado@ctm.com.es, postal address: Avda. de les

Bases de Manresa 1, 08242 Manresa, Spain, Telf.: +34938777373, Fax: +3493877737

\begin{abstract}
In this work, the applicability of the two most commonly used equations for calculating the fracture toughness by nanoindentation is discussed in terms of the indenter geometry and the indentation crack morphology. These equations are calibrated for Berkovich and cube-corner indenters taking into account the actual indentation crack morphology, aimed at attaining a more reliable estimation of fracture toughness in small material volumes by nanoindentation.
\end{abstract}

Keyworks: nanoindentation, fracture toughness, cube-corner indenter

Mechanical characterization of micro-volume systems as thin films [1,2] or micro-sized phases [3] is a critical step for optimizing and developing materials and high performance coatings. Nanoindentation has proved to be a suitable technique for the mechanical characterization of micro-sized material volumes in terms of hardness $(H)$, Young's modulus $(E)$ and fracture toughness in mode I $\left(K_{I C}\right)$. While $H$ and $E$ are measured from load-displacement curves [4], $K_{I C}$ is estimated from the length of indentation cracks following the indentation microfracture method (IM). It is based on the formation of cracks at the indentation corners when sharp indenters are applied. Such indentation cracks extend driven by the residual stress field up to a length that can be related to the material crack propagation resistance. Indentation, as a technique for measuring fracture toughness, was proposed in the 70's by Evans and Charles who related the stress intensity factor $(K)$ for Vickers indentation cracks to the observed crack lengths [5]. Since then, many expressions to compute $K_{I C}$ by indentation have been proposed [6]. Two of the most used are the equations proposed by Anstis et al [7] and by Laugier [8]. Both equations are based in the Lawn et al. extension of the Evans and Charles analysis [5]. Lawn et al, by using Hill's expanding cavity solution for an elastic-plastic solid and assuming a half-penny crack configuration, suggested the following expression to calculate $K_{I C}[5]$ : 


$$
K_{I C}=\xi_{R}\left(\frac{E}{H}\right)^{1 / 2} \frac{P}{c^{3 / 2}}
$$

Where $E$ is the Young's modulus, $H$ is the hardness, $P$ is the indentation load and $2 c$ is the total crack length (that is, $2 c=2 \ell+2 a$, where $\ell$ is the length of the crack from the indentation corner, and $2 \boldsymbol{a}$ is the indentation diagonal). The term $\xi_{R}$ is a material constant containing elements related to the geometry of the indenter and the morphology of the crack system that was considered to be halfpenny. In 1981, Anstis et al. fitted equation (1) by using a number of brittle materials with a well known $K_{I C}$, ranging from $0.74 \mathrm{MPa} \cdot \mathrm{m}^{1 / 2}$ (soda lime glass) to $12 \mathrm{MPa} \cdot \mathrm{m}^{1 / 2}$ (cemented WC-Co) [7]. Using the experimental values of $E, H, K_{I C}$, and $P / c^{3 / 2}$ for each material, the value of $\xi_{R}$ was found to be 0.016 . This value is well established when half-penny cracks are generated with Vickers indenters in the range of macro and microindentation. On the other hand, for superficial cracks as Palmqvist crack profiles, in 1987 Laugier adapted the Lawn halfpenny formalism and modified $\mathrm{K}$ to take into account the actual crack morphology [8],

$$
K_{I C}=\chi_{\nu}\left(\frac{l}{a}\right)^{-1 / 2}\left(\frac{E}{H}\right)^{2 / 3} \frac{P}{c^{3 / 2}}
$$

The symbols $E, H, P, a$ and $\ell$ have the same meaning as in equation (1). The fit of the equation for radial cracks leads to a value for the constant $\chi_{v}$ of 0.015 [8]. In spite of serious criticisms to the IM method for the determination of fracture toughness [9], it is still widely used as a simple, low-time consuming and inexpensive method. However, it is well known that if the constants $\xi_{R}$ and $\chi_{v}$ are not properly calibrated, the accuracy of the obtained values of $K_{I C}$ is low. Such constants were experimentally fitted by Anstis et al. and Laugier, respectively, using 4-sided Vickers pyramid indenters (in the range of macro and microindentation with applied loads up to $100 \mathrm{~N}$ and up to $1000 \mathrm{~N}$ in the case of cemented WC-Co) and they are well accepted and used. For 3-sided indenters, as Berckovich ones, less information is available in this high indentation load range. In 1992, Dukino et al. fitted Laugier equation (equation 2) including a modifying factor that accounts for the number of symmetric cracks proposed by Ouchterlony due to the nonsymmetrical nature of Berkovich indenters, obtaining $\chi_{v}=0.016[10]$.

Mechanical characterization of small volumes, such as thin films or micro-sized phases, has recently received major attention, and many works focuses on the determination of $K_{l C}$ by nanoindentation following the IM method [3, 11-16]. Nanoindentation allows applying low loads needed for accurate micromechanical characterization with high spatial resolution. However, the first point to consider when extending the applicability of IM to the nanoindentation load range is that the most used indenters in this technique are Berkovich and cube-corner instead of Vickers. Consequently, the fitted $\xi_{R}$ and $\chi_{\nu}$ parameters for Vickers indenters must be revised when Berkovich and cube-corner indenters are used since its dependence on indenter geometry and crack morphology should change the fitted values given by Vickers indenters. 
Sharper indenters than Berkovich or Vickers indenters, as the cube-corner one, displace a much larger volume of material for a given load. Therefore, higher stresses are induced beneath the indenter, thus, cracks can be generated at lower loads [12]. It allows characterizing even smaller material volumes $[12-13,17]$. The morphology of the indentation cracks depends on the indentation load, tip geometry and material toughness. Half-penny morphology, in which cracks are connected underneath the hardness impression, is the most common when testing brittle materials with Vickers indenters and with $c / a$ larger than 3 , while radial cracks are developed at low indentation loads or/and in materials with higher toughness, independently of the type of indenter, and with c/a smaller than 2.5 [18].

Accurate $K_{I C}$ measurements require a proper knowledge of the crack morphology in order to use the most appropriate expression for evaluating $K_{l C}$. From a phenomenological point of view equation (1) was developed to be used when half-penny cracks are generated, whereas equation (2) was adapted to describe Palmqvist cracks. Despite the number of works addressed to the evaluation of fracture toughness by nanoindentation, few of them deal with the accuracy of the different expressions for cube-corner indenters [14]. Moreover, $K_{I C}$ is often evaluated by nanoindentation using equations (1) or (2), among others, without considering the crack morphology and the used indenter tip. Thus, the aim of the present work is to assess the applicability of the IM method for obtaining $K_{I C}$ of small material volumes by means of nanoindentation, taking into account the influence of the indenter geometry and the generated crack morphology. The constants $\xi_{R}$ and $\chi_{\nu}$ of equations (1) and (2) will be fitted by testing materials with well known $K_{I C}$.

The materials used in this work have well known and accepted values of $K_{I C}$, obtained by standardized procedures, such as the SEPB, CNB or SCF methods, which do not precise experimental calibrations. The fracture toughness of the selected materials must not depend on the crack size, i.e. materials should not present an R-curve behavior. As cracks lengths generated by nanoindentation range from 1 to $9 \mu \mathrm{m}$ in single crystals, and up to $30 \mu \mathrm{m}$ in soda lime glass, if an R-curve behavior is present, the toughness developed with such cracks will differ from that obtained from large cracks and calibration using standardized methods will not give accurate results. Accordingly, the following three materials have been chosen: a) soda-lime glass; b) Si (100) single crystal; and c) SiC-6H (0001) single crystal. Table 1 shows the reported $K_{I C}$ values obtained by the SENB method for these materials. In order to have more data in the experimental calibration of $\chi_{v}$ for a cube-corner tip geometry, two additional single crystals were analyzed, $\mathrm{Si}(111)$ and $\mathrm{SiO}_{2}$ (0001). To our knowledge $K_{I C}$ obtained with standardized tests are not available for these two single crystals. To overcome this lack of knowledge $K_{I C}$ was determined by IM using equation (2) with $\chi_{v}=0.022$ (this value was obtained after calibration with soda-lime glass, $\mathrm{Si}(100)$ and $\mathrm{SiC}-6 \mathrm{H}$ (0001) for the Berkovich indenter, see table 2). 
Berkovich and cube-corner indenters were used in a XP Nanoindenter (MTS Corp.) with applied loads in the range of $200 \mathrm{mN}$ to $3 \mathrm{~N}$. After indentation, all the hardness impressions and generated crack morphology were imaged using a field-emission scanning electron microscope (FE_SEM) (Carl Zeiss SMT Inc.) to measure the sizes of the contact impression and the lengths of the cracks emerging from their corners. Crack morphology was examined by means of focus ion beam (FIB/SEM, Carl Zeiss Neon 40). An ion beam of 200 pA and $30 \mathrm{kV}$ was used; SEM images were taken every two ion beam polishing steps and reconstructed by using Avizo software. $\mathrm{H}$ and $\mathrm{E}$ were evaluated using the Oliver and Pharr methodology [4] performing Berkovich indentation tests, as both are essentially independent of the indenter used [11].

The indented materials showed a well defined crack pattern in the range of applied loads. Only indentations showing well developed cracks and without chipping, were used to calculate $K_{I C}$. Figure 1 shows a FIB tomography corresponding to a $100 \mathrm{mN}$ cube-corner indentation on a soda-lime glass sample, where three cracks emanating from the centre of the impression can be observed. Additionally, depending on the indentation load and the tip geometry, lateral cracks were also present. Tomography observations indicate that cracks generated by Berkovich and cube-corner indenters in the three evaluated materials are radial, with a semielliptical shape. Accordingly, form a geometrical point of view they are not semi-circular and not joint between them passing beneath the indentation impression as it is usually happens in high indentation load range with Vickers indenters. Nevertheless, both constants, $\xi_{R}$ and $\chi_{v}$ from equations (1) and (2), respectively, were determined for the Berkovich indenter, by fitting such equations to the experimental values of $H$ and $E, P$, reported value of $K_{I C}$ for each material (Table 1), and mean crack length (see Figure 2). The constant $\xi_{R}$ of equation (1) was determined even though this equation was not developed for radial cracks.

The values of $\xi_{R}$ and $\chi_{v}$ for Berkovich and cube-corner indenter geometries were experimentally fitted as $\xi_{R}=0.026$ and $\chi_{v}=0.022$ or 0.057 depending on the indenter geometry (Table 2). Equation (2) presents the best fit for Berkovich indenters (Figure 2). According to this and because cube-corner indenters give radial cracks, equation (2) were only use to fit the experimental data obtained with the cube-corner tip (Figure 3), giving rise to good results. The calculated values of $K_{I C}$ (using the proposed $\chi_{v}$ ) for both type of indenters are close to the accepted $K_{I C}$ values for the tested materials (obtained by the SENB method (Table 1)). This good fitting can be rationalized considering the morphology of the indentation crack. Equation (2) was developed for radial cracks with semielliptical shape, the same as the experimentally identified cube-corner cracks (with also semielliptical shape, figure 1). The effect of the proper selection of the equation to calculate $K_{I C}$ is showed when calculating fracture toughness with cube-corner indenters. Equation (2) with $\chi_{v}=0.015$ (obtained by Laugier for a Vickers indenter) gives a $K_{I C}$ for $\mathrm{Si}(100)$ of $0.31 \pm 0.04 \mathrm{MPam}^{1 / 2}$ (calculated in reference 13). Whereas if the calibrated $\chi_{v}$ for the cube-corner indenter is used $\left(\chi_{v}=0.057\right), K_{I C}$ is $1.0 \pm 0.1$, which is much close to the accepted $K_{I C}$ for this material (see Table 2). Thus, it is clear that a proper calibration 
of experimental equations is crucial to obtain accurate values of $K_{I C}$ by nanoindentation when using Berkovich and cube-corner indenters.

The obtained calibration for cube-corner indenter differs from that obtained in previous works $[10-11,13,15,19-20]$. A range of values from 0.033 to 0.040 are reported for cube-corner indenters [11, 13, 15, 19-20], whereas in this work a value of 0.057 has been obtained. If it is assumed that the parameter is related to tip angle only a value of 0.033 can be expected. On the other hand, depending on the toughness range of the materials studied, a value of 0.0319 [19], 0.036 [11] or 0.040 [20] is reported. The main reason for the discrepancy observed between the literature values and the values given in this work is the chosen equation to fit the experimental data. In the literature, for cube-corner indenters, equation (1) was fitted. But, in this work it is shown that the crack morphology has a semielliptical shape, which indicates that from a phenomenological point of view equation (2) should be used to fit the results. With respect to Berkovich indenters, Dukino et al. reported $\chi_{v}=0.016$ (fit performed in the microindentation range, up to $5 \mathrm{~N}$ ), where the here obtained value is slightly different, 0.022 [10]. Such discrepancy could come from the different formulation of the used equations as discussed above.

Concerning the applicability range of the proposed values for $\xi_{R}$ and $\chi_{v}$, this, will be within the fracture toughness values studied, i.e., at least up to $3.4 \mathrm{MPa} \cdot \mathrm{m}^{1 / 2}$. It covers a wide range of glasses and traditional and engineering ceramics.

The experimental calibration of $\xi_{R}$ and $\chi_{v}$ in this work has been performed using a soda-lime glass and some single crystals. Referring to the later, crystal anisotropy must be considered when indenting single crystals, since it is known that indentation cracking depends on the crystallographic orientation. In this sense, fracture toughness measurement may be specially sensitive to the relative crystallographic planes-indenter tip orientation. Ebrahimi et al. studied the fracture anisotropy of Si single crystal with Vickers tips, indenting different crystallographic planes and considering the fracture along different crystallographic directions [21]. They show a clear influence of the crystal anisotropy on the fracture toughness. However, $K_{I C}$ remains almost constant when indenting the (001) and (111) planes, but large scatter is found when indenting the (110) plane. [21] Thus, aimed at minimizing the effect of crystal anisotropy on indentation cracking and getting reliable values of $\xi_{R}$ and $\chi_{v}$, Si (100) and Si (111) single crystals were chosen to calibrate equations (1) and (2). It has also been experimentally assessed by carefully revising the crack pattern in the tested single crystals, without evidences of preferred fracture directions or abrupt changes in the crack propagation direction for different crystal-tip orientations. Additionally, crack lengths do not vary significantly; at least the variations are similar to those measured in the amorphous and isotropic sample (soda-lime glass). Such results show that crystal anisotropy does not significantly affect the values of $\xi_{R}$ and $\chi_{v}$ reported in this work. Nevertheless, crystal anisotropy must be kept in mind when characterizing single 
crystals by nanoindentation, and accurate revision of crack patterns is highly recommended as well as considering the relative orientation of the indenter tip in the indented plane.

In summary, examination of the crack morphology shows that indentation cracks are radial with a semielliptical shape when Berkovich and cube-corner indenters are used in nanoindentation. Therefore, from a phenomenological point of view Laugier's equation would be more adequate for evaluating $K_{l C}$. This equation has been calibrated obtaining a calibration constant $\chi_{v}$ of 0.022 \pm 0.001 for a Berkovich indenter and $0.057 \pm 0.002$ for a cube-corner indenter, which gives nanoindentation fracture toughness close to the actual fracture toughness measured by standardized methods in materials without R-curve behavior.

[1] Chen, J., Bull, S.J., J. Phys. D.: Appl. Phys. 40 (2007) 5401.

[2] Malzbender, J., den Toonder, J.M.J, Balenende, A.R., de With, G, Mat. Sci. and Eng. R 36 (2002) 47.

[3] Casellas, D., Caro, J., Molas, S., Prado, J.M., Valls, I., Act. Mater. 55 (2007) 4277.

[4] Oliver, W.C., Pharr, G.M., J. Mater. Res. 7 (1992) 1564.

[5] Lawn, B.R., Evans, A.G., Marshall, D.B., J. Am. Ceram. Soc. 63 (1980) 574.

[6] Ponton, C.B., Rawlings, R.D., Mater. Sci. Tech. 5 (1989) 865

[7] Anstis, G.R., Chantikul, P., Lawn, B.R., Marshall, D.B., J. Am. Ceram. Soc. 64 (1981) 533.

[8] Laugier, M.T., J. Mater. Sci. Letters 73 (1987) 897.

[9] Quinn, G. D., Bradt, R. C., J. Am. Ceram. Soc., 90 [3] (2007) 673 - 680.

[10] Dukino, R.D., Swain, M.V., J. Am. Ceram. Soc. 75 (1992) 3299.

[11] Harding, D.S., Oliver, W.C., Pharr, G.M., Mat. Res. Soc. Symp. Proceed. 356 (1995) 663.

[12] Jang, J., Pharr, G.M., Acta Materialia 56 (2008) 4458.

[13] Morris, D.J., Cook, R.F., J. Am. Ceram. Soc. 87 (2004) 1494.

[14] Schiffmann, K.I., Phil. Mag. 91 (2011) 1163.

[15] Bull, S.J., Comp. Rend. Mec. 339 (2011) 5178.

[16] Jungk, J.M., Boyce, B.L., Buchheit, T.E., Friedmann, T.A., Act. Mater. 54 (2006) 4043.

[17] Zhang, T., Feng, Y., Yang, R., Jiang, P., Scrip. Mater. 62 (2010) 199.

[18] Schneider G. A. and Fett T., J. Ceram. Soc. Japan, 114[11], 1044-48 (2006).

[19] Pharr, G.M., Harding, D.S., Oliver, W.C., Dordrecht, Kluwer (1993) 449.

[20] Pharr, G.M., Mater. Sci. Eng. A-Struct. Mater. Prop. Microstruct. Process. 253 (1998) 151.

[21[ Ebrahimi, F., Kalwani, L., Mat. Sci. and Eng. A268 (1999) 116.

[22] Henshall, J.L., Brookes, C.A., J. Mat. Lett. 4 (1985) 783.

[23] Xin, Y.B., Hsia, K.J., Lange, D.A., J. Am. Ceram. Soc. 78 (1995) 3201.

$$
\mathrm{K}_{\mathrm{lc}}\left(\mathrm{MPa} \cdot \mathrm{m}^{1 / 2}\right)
$$




\begin{tabular}{|c|c|c|c|c|}
\hline Material & SENB & $\begin{array}{c}\text { Berkovich } \\
\text { Anstis et al. }\end{array}$ & $\begin{array}{c}\text { Berkovich } \\
\text { Laugier }\end{array}$ & $\begin{array}{c}\text { Cube-corner } \\
\text { Laugier }\end{array}$ \\
\hline $\mathrm{SiC}-6 \mathrm{H}(0001)$ & $3.4^{[22]}$ & $2.3 \pm 0.1$ & $3.0 \pm 0.3$ & $3.5 \pm 0.8$ \\
\hline $\mathrm{Si}(100)$ & $0.95^{[23]}$ & $0.9 \pm 0.2$ & $1.0 \pm 0.3$ & $1.0 \pm 0.1$ \\
\hline Soda-lime glass & $0.7^{[/]}$ & $0.6 \pm 0.1$ & $0.6 \pm 0.2$ & $0.8 \pm 0.1$ \\
\hline $\mathrm{SiO}_{2}(0001)$ & & & $1.1 \pm 0.1$ & $1.0 \pm 0.1$ \\
\hline $\mathrm{Si}(111)$ & & & $1.4 \pm 0.3$ & $1.8 \pm 0.2$ \\
\hline
\end{tabular}

Table 1. $\mathrm{K}_{\mathrm{IC}}$ comparison using Anstis et al. equation and Laugier equation with the different calibrations of $\xi_{\mathrm{R}}$ and $\chi_{\mathrm{U}}$ reported in Table 3.

\begin{tabular}{|c|c|c|c|}
\hline \multirow{2}{*}{ Indenter's geometry } & \multirow{2}{*}{ Crack morphology } & \multicolumn{2}{|c|}{ Equation } \\
\cline { 3 - 4 } & & Anstis et al. & Laugier \\
\hline Vickers & Half-penny & $\xi_{R}=0.016$ & \\
\hline Vickers & Radial ( Palmqvist) & & $\chi_{v}=0.015$ \\
\hline Berkovich & Radial (Palmqvist) & $\xi_{R}=0.026 \pm 0.001$ & $\chi_{v}=0.022 \pm 0.001$ \\
\hline Cube-corner & Radial ( Palmqvist) & & $\chi_{v}=0.057 \pm 0.002$ \\
\hline
\end{tabular}

Table 2. Experimental calibration of the constants $\xi_{R}$ and $\chi_{\nu}$ for a Berkovich and cube-corner indenters for Anstis et al. (equation (1)) and Laugier (equation (2)) equations.

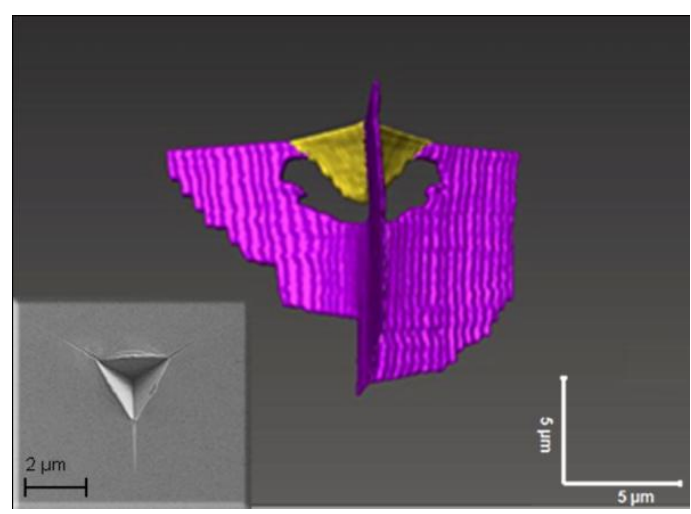

Figure 1. FIB tomography for a cube-corner indenter at $100 \mathrm{mN}$ in a soda-lime glass sample.

Radial cracks with semielliptical shape can be discerned. Yellow area corresponds to the indentation impression, violet indicates the crack morphology. 


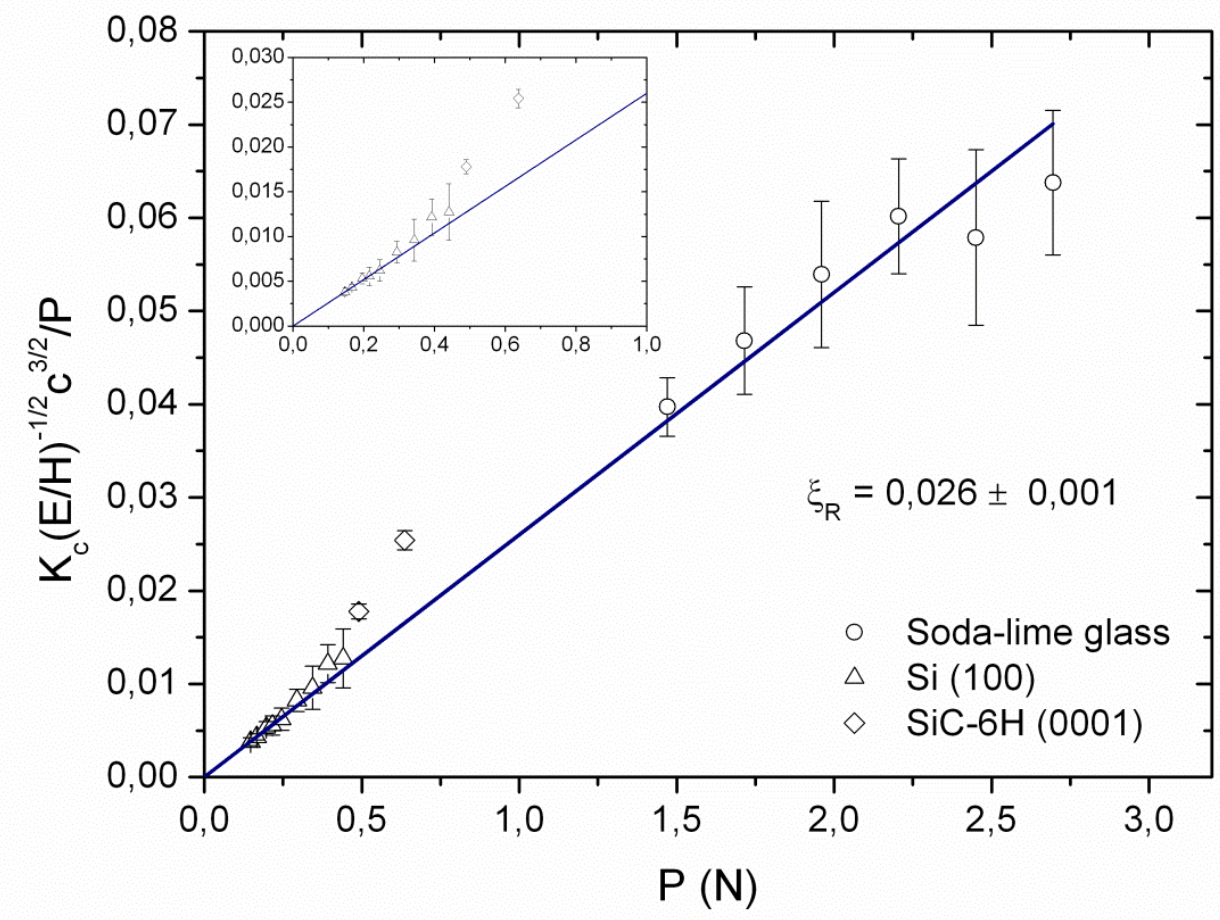

(a)

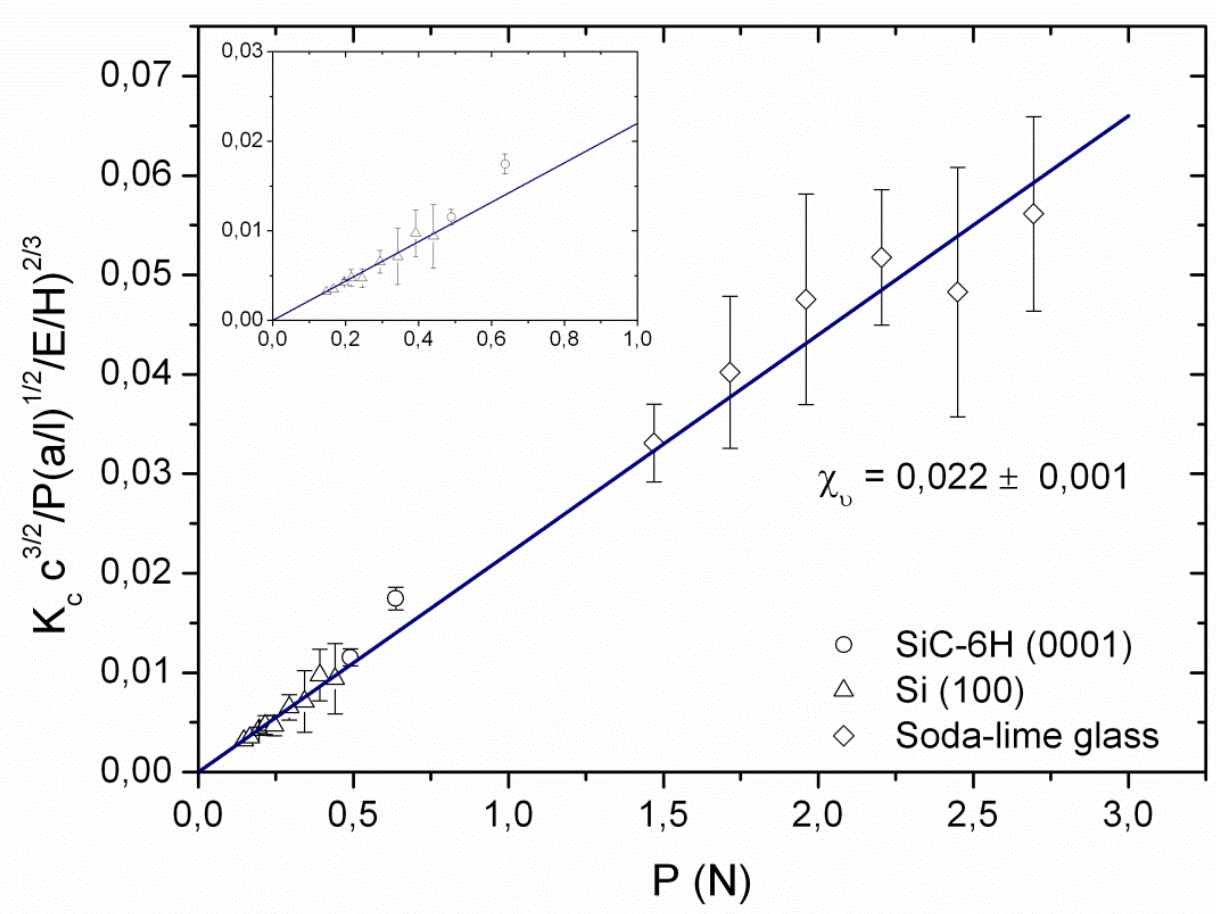

(b)

Figure 2. Calibration of the constants $\xi_{\mathrm{R}}$ and $\chi_{\mathrm{v}}$ for a Berkovich indenter in (a) Anstis's equation (Eq. (1)) and (b) Laugier's equation (Eq. (2)). 


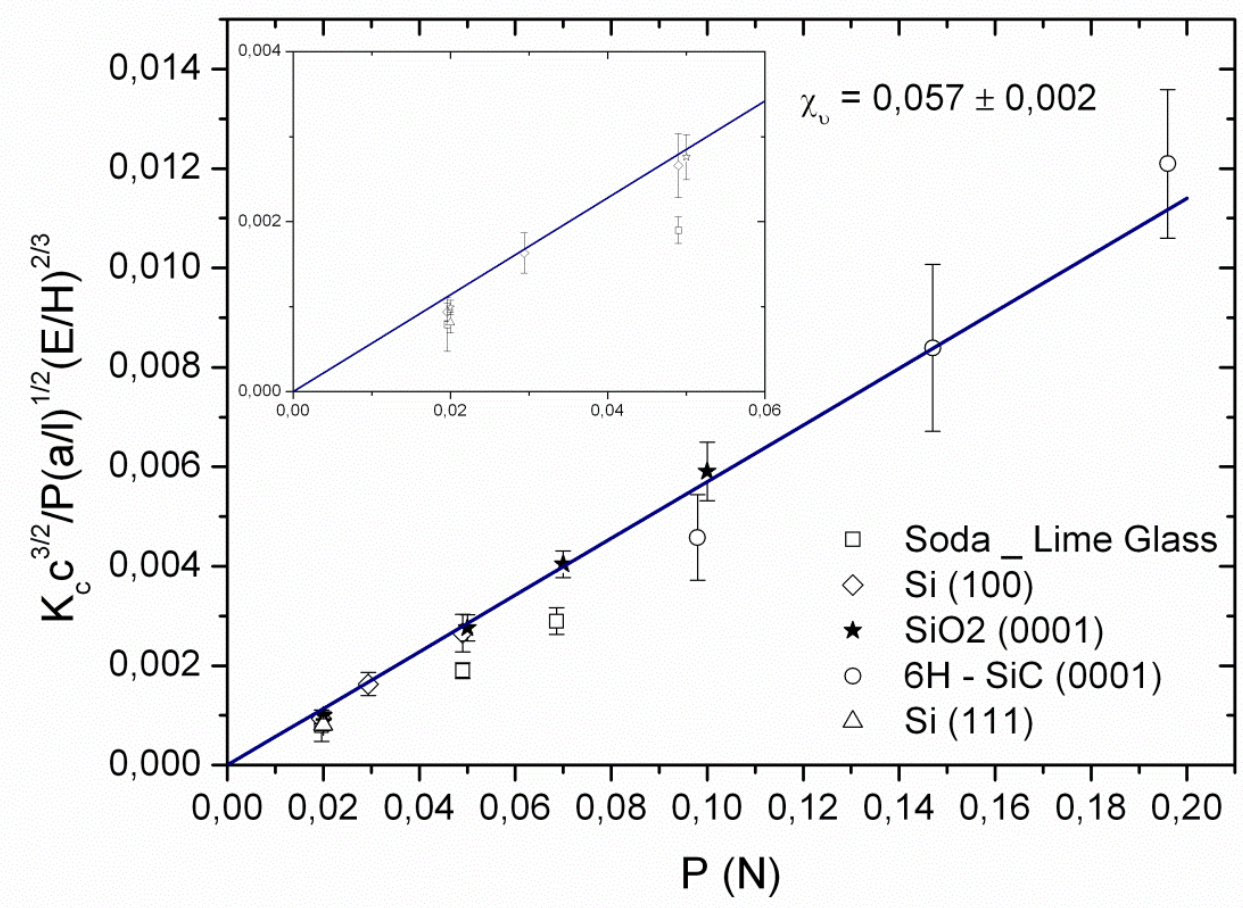

Figure 3. Calibration of the constant $\chi_{v}$ for a cube-corner indenter in Laugier's equation (Eq. (2)). 